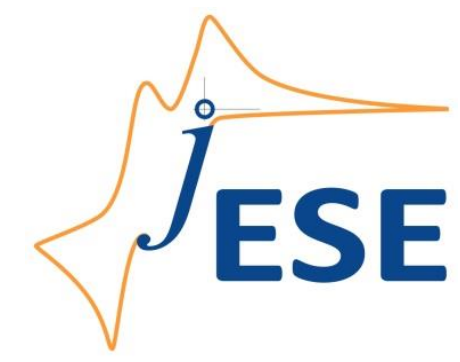

Open Access : : ISSN 1847-9286

www.jESE-online.org

Original scientific paper

\title{
Electrochemical studies on zirconium phosphoborate based heterogeneous membranes
}

\author{
SANDEEP KAUSHAL, PRITPAL SINGH* AND SUSHEEL K. MITTAL**, \\ Department of Applied Sciences and Humanities, Punjab Technical University, Jalandhar, India \\ * Sri Guru Granth Sahib World University, Fatehgarh Sahib (Pb), India \\ **School of Chemistry and Biochemistry, Thapar University, Patiala 147004, India \\ Corresponding Author: E-mail: smittal@thapar.edu; Fax: +91-175-2364498
}

Received: September 30, 2013; Revised: March 18, 2014; Published: May 13, 2014

\begin{abstract}
Electrode potential measurements have been applied to study electrical characteristics like transport numbers, permselectivity \& fixed charged density of zirconium phosphoborate ion exchange membranes. The potential measurements were made across the cation exchange membrane maintained at $27 \pm 0.1^{\circ} \mathrm{C}$, using halide and nitrate salts of alkali and alkaline earth metals as electrolytes. The membrane potentials, transport numbers and permselectivity values increase with increase in average concentration from $0.0055 M$ to $0.0495 M$ for 1:1 and 1:2 electrolytes. With the increase in concentration of the electrolyte, the number of counter ions interacting with the membrane surface increases leading to enhanced Donnan exclusion responsible for the increase of transport numbers. Fixed charge density of the membrane $(X)$ for 1:2 electrolytes is higher in magnitude than for 1:1 electrolytes indicating that the cation exchange is taking place as hydrated species. This hypothesis is supported by higher transport numbers for alkaline earth metal ions than alkali metal ions throughout the range of concentration.
\end{abstract}

\section{Keywords}

Electrochemical studies, lon-exchange membrane, transport numbers, alkali metal ions, ZrPB

\section{Introduction}

Inorganic ion-exchangers are stable towards chemical degradation and are more selective for metal ions over their organic analogs [1]. The ion-exchange membranes have diverse applications, from desalting of brackish water to treating industrial effluents as well as processing biological effluents. Due to the development of new ion-exchange membranes with better selectivities, lower electrical resistance and improved electrochemical and chemical properties, the ion 
exchange membranes find applications in food, drugs, chemical processes and biotechnology industries $[2,3]$. The great interest in the ion exchange membranes is mainly due to their exceptional electrochemical properties.

The ion exchange membranes combine the ability to act as a separation wall between the two solutions, with chemical and electrochemical properties of ion exchanger. The most important of these are the pronounced difference in permeability for counter ions, co-ions and neutral molecules and their high electrical conductivity. When in contact with electrolyte solutions of low or moderate concentrations, the membrane contains a large number of counter ions but relatively few co-ions, due to Donnan exclusion. The membrane is perm selective for counter ions. The perm selectivity is reflected not only in differences in permeability, but also in electrical potential difference which arises between the two solutions (membrane potential).

The effect of membrane potential and adsorption on the permeability of ions, electrical conductance, ion exchange capacity and perm selectivity behavior on diffusion phenomena in a large number of inorganic membranes was studied by Malik et. al. [4-9]. Gnusin et. al. [10] investigated the concentration dependence of a wide range of transport characteristics of inorganic ion exchange membranes. To assess the suitability of any membrane for any specific application, two vital parameters i.e. the transport characteristics and its structural properties must be evaluated. The behavior of ion exchange membranes (IEMs) depends on the physiochemical properties, in addition to the nature of electrolyte solutions used. It is also beneficial to predict the behavior of prepared membranes on the basis of their structural properties. Few reports are available on investigation of the effect of nature of electrolyte solutions' on IEMs properties despite the fact that they have significant influences [11-16].

In this paper, electrode potential measurements have been used to study electrical characteristics like transport numbers, perm selectivity and fixed charge density across zirconium phosphoborate based heterogeneous ion exchange membranes. Halide and nitrate salts of alkali and alkaline earth metals were used as electrolytes. The effect of electrolyte concentration on transport numbers and perm selectivity on the ion exchange characteristics of zirconium phosphoborate membrane has also been studied. The membrane behaved as cation selective under ambient experimental conditions. The counter ion transport number, membrane perm selectivity and concentration of fixed ionic sites in the zirconium phosphoborate membrane are estimated by membrane potential data. Fixed ionic concentration data have been analyzed in the light of non-thermodynamic principle for its correlation with membrane structure and permeability behavior. The proposed membrane system has been used to study the selective behavior for the alkali/ alkaline earth metal ions.

\section{Experimental}

\section{Reagents}

Zirconyl oxychloride (Loba Chemie, India), boric acid (S.D. Fine Chem., India) and phosphoric acid (S.D. Fine Chem., India) were used for synthesis. All other chemicals used were of A.R. grade. Standard solutions were prepared by direct weighing of AR grade reagents using double distilled water.

\section{Synthesis of zirconium phosphoborate}

The exchanger was prepared by adding zirconyl oxychloride $(0.1 \mathrm{M})$ solution to a continuously stirred mixture of boric acid solution $(0.1 \mathrm{M})$ and phosphoric acid solution $(0.1 \mathrm{M})$ at $60^{\circ} \mathrm{C}$, in the 
volume ratio 2:1:1. The gel produced was allowed in round bottomed flask to stand overnight. Then the gel was repeatedly washed with distilled water to remove chlorides from the mother liquor. The absence of chlorides in the mother liquor was tested with $\mathrm{AgNO}_{3}$ solution. After the gel became free from chlorides, it was filtered through Whatmann No.1 filter paper using Buchner funnel and suction pump. The gel was transferred from Buchner funnel to petri dish. The precipitates in the Petri dish were dried in an air oven at $40^{\circ} \mathrm{C}$. When the gel dried completely, distilled water was added. Small granules of the ion-exchanger were formed with cracking sound.

\section{Determination of ion-exchange capacity}

lon-exchange capacity was determined by column operation. Exchanger in the $\mathrm{H}^{+}$-form was placed in a column containing a glass wool support. Sodium nitrate solution (1.0 M) was used as an eluent and about $400 \mathrm{ml}$ of it was passed at a rate of 8-10 drops per minute through the ion exchanger column containing $1 \mathrm{~g}$ of exchanger. $\mathrm{H}^{+}$eluted from the column was determined titrimetrically against standard solution of sodium hydroxide.

\section{Preparation of Membrane}

A desired quantity of the finely ground zirconium(IV) phosphoborate (ZrPB) was added to epoxy resin in the ratio ZrPB : epoxy resin $=80: 20(\mathrm{w} / \mathrm{w})$ with constant stirring till a homogeneous slurry was obtained. This slurry was spread between the folds of a filter paper and dried in air to get the membrane of $\cong 1 \mathrm{~mm}$ thickness. The dried membrane was dipped in distilled water to remove the filter paper. The sheet of the membrane thus obtained was cut with a sharp knife into circular discs of about $18 \mathrm{~mm}$ diameter. The membrane discs with good surface qualities were selected for further investigations

\section{Electrode Assembly}

The membrane was pasted on one end of the electrode assembly using araldite. The membrane was fixed from backside to other part of the electrode assembly. The electrode chambers were filled with $1.0 \mathrm{M}$ solution of each of the electrolytes such as lithium chloride, sodium chloride, potassium chloride, magnesium chloride and barium chloride for 16 hours, to convert it into appropriate ionic form. After equilibration, the electrode assembly was washed with demineralized water (DMW). The membrane was kept immersed in DMW for 2 hours to remove the excess of electrolyte solution. When not in use, the electrode chambers were filled with demineralized water. The electrode assembly was kept immersed in water thermostat maintained at $27 \pm 0.1{ }^{\circ} \mathrm{C}$. Membrane potential measurements were made using saturated calomel electrodes as reference electrodes.

\section{$\mathrm{Ag} / \mathrm{AgCl}(\mathrm{s}), \mathrm{Cl}^{-}||$solution $\mathrm{C}_{1} \mid$ Membrane|solution $\mathrm{C}_{2}|| \mathrm{Cl}^{-}, \mathrm{AgCl}(\mathrm{s}) \mid \mathrm{Ag}$}

Potential measurements were made for different concentrations of the same electrolyte on two sides of the membrane in such a way that the concentration ratio $C_{2} / C_{1}=10$. The potential difference developed across the membrane was measured against $\mathrm{Ag} / \mathrm{AgCl}$ reference electrode on either side of membrane, using a digital potentiometer. The electrode chambers were rinsed with electrolyte solution of next higher concentration and then filled with the same solution. The membrane was allowed to equilibrate for 2 hours and the new potential difference was then noted.

The membrane potentials across zirconium phosphoborate membrane were determined using some 1:1 electrolytes such as lithium chloride, sodium chloride, potassium chloride, sodium 
nitrate, lithium nitrate and potassium nitrate and some 1:2 electrolytes such as magnesium chloride, calcium chloride, strontium chloride, barium chloride, magnesium nitrate, calcium nitrate strontium nitrate and barium nitrate, in overall concentration range of $0.001 \mathrm{M}$ to $0.1 \mathrm{M}$. The membrane potential measurements were reproducible to $\pm 0.1 \mathrm{mV}$.

\section{Results and Discussion}

\section{Membrane Potentials}

A membrane separating electrolyte solutions of unequal concentrations exhibits a difference in electrical potential due to unequal ionic mobility and is equal to liquid junction potential in a nonselective membrane. In an ideally selective membrane, according to the TMS theory [17], the membrane potential values are related to the activities of the electrolyte on the two sides of membrane, $a_{1}$ and $a_{2}$ :

$$
E_{m}=2.303 \frac{R T}{n F}\left(2 \bar{t}_{+}-1\right) \log \frac{a_{2}}{a_{1}}
$$

where $E_{\mathrm{m}}$ is membrane potential across the membrane.

The maximum electrical potential is given by:

$$
\left[\left(E_{\mathrm{m}}\right)_{\mathrm{t}=0}\right]_{\max }=\frac{R T}{n F} \ln \frac{a_{2}}{a_{1}}
$$

provided the solutions are dilute. $E_{\mathrm{m}}$ changes with change in mean concentration of the electrolyte. From Table 1 and 2, it is evident that higher membrane potentials are observed at higher concentrations of electrolytes and membrane potential increases with increase in concentration of the electrolyte. For 1:1 halide electrolytes, membrane potentials are in order

$$
\mathrm{Li}^{+}>\mathrm{K}^{+}>\mathrm{Na}^{+}
$$

Table 1: Membrane potential values of 1:1 electrolytes for zirconium phosphoborate

\begin{tabular}{ccccccc}
\hline \multirow{2}{*}{$\begin{array}{c}\text { Concentration of } \\
\text { electrolyte, } \mathbf{M}\end{array}$} & $\mathrm{LiCl}$ & $\mathrm{NaCl}$ & $\mathrm{KCl}$ & $\mathrm{LiNO}_{3}$ & $\mathrm{NaNO}_{3}$ & $\mathrm{KNO}_{3}$ \\
\cline { 2 - 7 } & 0.02 & 0.012 & 0.02 & 0.01 & 0.026 & 0.016 \\
\hline $0.001-0.01$ & 0.03 & 0.016 & 0.02 & 0.016 & 0.026 & 0.018 \\
\hline $0.002-0.02$ & 0.04 & 0.017 & 0.02 & 0.018 & 0.027 & 0.022 \\
\hline $0.003-0.03$ & 0.04 & 0.019 & 0.02 & 0.019 & 0.031 & 0.023 \\
\hline $0.004-0.04$ & 0.05 & 0.02 & 0.02 & 0.02 & 0.033 & 0.025 \\
\hline $0.005-0.05$ & 0.05 & 0.02 & 0.03 & 0.021 & 0.035 & 0.028 \\
\hline $0.006-0.06$ & 0.05 & 0.021 & 0.02 & 0.024 & 0.036 & 0.027 \\
\hline $0.007-0.07$ & 0.05 & 0.021 & 0.02 & 0.025 & 0.036 & 0.028 \\
\hline $0.008-0.08$ & 0.05 & 0.022 & 0.02 & 0.025 & 0.037 & 0.029 \\
\hline $0.009-0.09$ & 0.05 & 0.026 & 0.01 & 0.025 & 0.038 & 0.028 \\
\hline $0.01-0.1$ & & & & & &
\end{tabular}

This order of the membrane potentials prevails in the concentration range 0.01 to $0.06 \mathrm{M}$, while for 1:1 nitrates, membrane potentials are in the order:

$$
\mathrm{K}^{+}>\mathrm{Na}^{+}>\mathrm{Li}^{+}
$$


Table 2. Membrane potential values of 1:2 electrolytes for zirconium phosphoborate

\begin{tabular}{ccccccccc}
\hline $\begin{array}{c}\text { Concentration of } \\
\text { electrolyte, } \mathbf{M}\end{array}$ & \multicolumn{7}{c}{ Membrane potential, $\mathbf{~}$} \\
\cline { 2 - 9 } & $\mathrm{MgCl}_{2}$ & $\mathrm{CaCl}_{2}$ & $\mathrm{SrCl}_{2}$ & $\mathrm{BaCl}_{2}$ & $\mathrm{Mg}\left(\mathrm{NO}_{3}\right)_{2}$ & $\mathrm{Ca}\left(\mathrm{NO}_{3}\right)_{2}$ & $\mathrm{Sr}\left(\mathrm{NO}_{3}\right)_{2}$ & $\mathrm{Ba}\left(\mathrm{NO}_{3}\right)_{2}$ \\
\hline $0.001-0.01$ & 0.021 & 0.025 & 0.017 & 0.034 & 0.019 & 0.020 & 0.0165 & 0.019 \\
\hline $0.002-0.02$ & 0.028 & 0.032 & 0.026 & 0.041 & 0.027 & 0.026 & 0.027 & 0.026 \\
\hline $0.003-0.03$ & 0.033 & 0.035 & 0.031 & 0.042 & 0.032 & 0.031 & 0.032 & 0.030 \\
\hline $0.004-0.04$ & 0.036 & 0.038 & 0.034 & 0.044 & 0.035 & 0.034 & 0.035 & 0.031 \\
\hline $0.005-0.05$ & 0.038 & 0.040 & 0.036 & 0.045 & 0.038 & 0.035 & 0.036 & 0.032 \\
\hline $0.006-0.06$ & 0.040 & 0.041 & 0.038 & 0.046 & 0.040 & 0.036 & 0.037 & 0.033 \\
\hline $0.007-0.07$ & 0.041 & 0.042 & 0.039 & 0.046 & 0.042 & 0.037 & 0.038 & 0.034 \\
\hline $0.008-0.08$ & 0.042 & 0.043 & 0.040 & 0.047 & 0.042 & 0.038 & 0.040 & 0.034 \\
\hline $0.009-0.09$ & 0.042 & 0.043 & 0.40 & 0.047 & 0.042 & 0.038 & 0.040 & 0.035 \\
\hline $0.01-0.1$ & 0.043 & 0.042 & 0.038 & 0.048 & 0.041 & 0.039 & 0.039 & 0.036 \\
\hline
\end{tabular}

The ionic perm selectivity of membrane is also expressed quantitatively, based on migration of counter ions through cation exchange membrane [11,16,18-26]:

$$
P_{s}=\left(\bar{t}_{+}-t_{+}\right) /\left(1-t_{+}\right)
$$

Where $\bar{t}_{+}$refers to the value of transport number in the membrane and $t_{+}$is the transport number of counter ions in solution [27].

Ion exchange capacity of ZrPB

The ion exchange capacity of zirconium phosphoborate was determined for some monovalent and bivalent cations like $\mathrm{Na}^{+}, \mathrm{K}^{+}, \mathrm{Mg}^{2+}$ and $\mathrm{Ca}^{2+}$ cations and is given in Table 3 .

Table 3. Ion exchange capacity of zirconium phosphoborate for some alkali and alkaline earth metals

\begin{tabular}{cccc}
\hline Sr. No. & Metal ion & Salt solution used & lon-exchange capacity, eq kg-1 \\
\hline 1 & $\mathrm{Na}^{+}$ & $\mathrm{NaCl}$ & 0.29 \\
\hline 2 & $\mathrm{~K}^{+}$ & $\mathrm{KCl}$ & 0.58 \\
\hline 3 & $\mathrm{Ca}^{2+}$ & $\mathrm{CaCl}_{2}$ & 0.52 \\
\hline 4 & $\mathrm{Mg}^{2+}$ & $\mathrm{MgCl}_{2}$ & 0.32 \\
\hline
\end{tabular}

The ion exchange capacity of ZrPB has been found in the order $\mathrm{K}^{+}>\mathrm{Na}^{+}$and $\mathrm{Ca}^{2+}>\mathrm{Mg}^{2+}$. These results suggested that the ion exchange capacity decreases as the radii of hydrated metal ions increase.

The magnitude of membrane potential depends on:

1. Adsorption of anions of diffusing electrolyte on membrane surface

2. Exchangeability of cations

3. Size of cations

4. Diffusion potential across the membrane

Higher membrane potential for lithium is because of its small size and high positive field around it, hence, it establishes higher potential across the charged membrane. Higher the potential, higher is the permeability [28] as observed in our experiments in the concentration range (0.01 to 
$0.06 \mathrm{M})$. The trend in the membrane potential and hence greatest permselectivity for lithium ions is because ion mass transfer coefficient through the ion-exchange membrane increases as a function of ion size [29].

lonic radii and hydrated ionic radii

Smaller the size of the alkali metal less is the ionic radius. As the size increases from top to bottom, ionic radii increase. The extent of hydration depends upon the size of the ion. Smaller the size of the ion, more highly it is hydrated and greater is its hydrated ionic radius and less is its ionic mobility (conductance). The radii and mobility of alkali metal ions are given in table 4 .

Table 4. Values of ionic radii, hydration numbers, hydrated ionic radii and ionic mobility of alkali metal ions

\begin{tabular}{|l|c|c|c|c|c|}
\hline Metal ions & $\mathbf{L i}^{+}$ & $\mathbf{N a}^{+}$ & $\mathbf{K}^{+}$ & $\mathbf{R b}^{+}$ & $\mathbf{C s}^{+}$ \\
\hline lonic Radius, pm & 76 & 102 & 138 & 152 & 167 \\
\hline Hydration Number & 25.3 & 16.6 & 10.5 & 10.0 & 9.9 \\
\hline Hydrated Radius, pm & 340 & 276 & 232 & 228 & 228 \\
\hline lonic Mobility & 33.5 & 43.5 & 64.5 & 67.5 & 68 \\
\hline
\end{tabular}

\section{Transport Numbers}

The transport number in exchanger phase is calculated from the slope of equation (1). The transport numbers and perm selectivity values of 1:1 halides and nitrates, and the transport numbers of 1:2 halides and nitrates are given in the Table 5 and 6, respectively.

Table 5. Transport numbers and perm selectivity values at mean concentration for 1:1 electrolytes

\begin{tabular}{|c|c|c|c|c|c|c|c|c|c|c|c|c|}
\hline \multirow{3}{*}{$\begin{array}{l}\text { Mean concen- } \\
\text { tration of } \\
\text { electrolyte, } M\end{array}$} & \multicolumn{6}{|c|}{ Transport No. $\left(\bar{t}_{+}\right)$} & \multicolumn{6}{|c|}{ Perm selectivity $\left(\boldsymbol{P}_{\mathrm{s}}\right)$} \\
\hline & \multicolumn{3}{|c|}{$\begin{array}{l}\text { Metal halide } \\
\text { electrolyte }\end{array}$} & \multicolumn{3}{|c|}{$\begin{array}{l}\text { Metal nitrate } \\
\text { electrolyte }\end{array}$} & \multicolumn{3}{|c|}{$\begin{array}{l}\text { Metal halide } \\
\text { electrolyte }\end{array}$} & \multicolumn{3}{|c|}{$\begin{array}{l}\text { Metal nitrate } \\
\text { electrolyte }\end{array}$} \\
\hline & LiCl & $\mathrm{NaCl}$ & $\mathrm{KCl}$ & $\mathrm{LiNO}_{3}$ & $\mathrm{NaNO}_{3}$ & $\mathrm{KNO}_{3}$ & $\mathrm{LiCl}$ & $\mathrm{NaCl}$ & $\mathrm{KCl}$ & $\mathrm{LiNO}_{3}$ & $\mathrm{NaNO}_{3}$ & $\mathrm{KNO}_{3}$ \\
\hline 0.0055 & 1.08 & 0.53 & 0.75 & 0.45 & 1.15 & 0.8 & 1.12 & 0.23 & 0.51 & 0.179 & 1.247 & 0.609 \\
\hline 0.011 & 1.44 & 0.71 & 0.67 & 0.74 & 1.17 & 0.85 & 1.65 & 0.52 & 0.35 & 0.613 & 1.280 & 0.706 \\
\hline 0.0165 & 1.66 & 0.75 & 0.89 & 0.81 & 1.21 & 0.92 & 1.98 & 0.59 & 0.71 & 0.717 & 1.344 & 0.843 \\
\hline 0.022 & 1.85 & 0.86 & 0.93 & 0.05 & 1.4 & 0.94 & 2.26 & 0.77 & 0.86 & 0.777 & 1.656 & 0.882 \\
\hline 0.0275 & 2.02 & 0.89 & 0.97 & 0.92 & 1.47 & 0.97 & 2.51 & 0.82 & 0.94 & 0.881 & 1.771 & 0.941 \\
\hline 0.033 & 2.06 & 0.91 & 0.99 & 0.94 & 1.55 & 1.03 & 2.56 & 0.85 & 0.98 & 0.911 & 1.899 & 1.059 \\
\hline 0.0385 & 2.09 & 0.93 & 0.98 & 1.1 & 1.6 & 1.01 & 2.6 & 0.89 & 0.96 & 1.147 & 1.979 & 1.02 \\
\hline 0.044 & 2.14 & 0.95 & 1.01 & 1.1 & 1.62 & 1.04 & 2.67 & 0.91 & 1.02 & 1.147 & 2.01 & 1.078 \\
\hline 0.0495 & 2.16 & 0.96 & 1.02 & 1.12 & 1.66 & 1.05 & 2.7 & 0.93 & 1.04 & 1.176 & 2.075 & 1.098 \\
\hline 0.055 & 2.22 & 1.2 & 0.39 & 1.13 & 1.71 & 1.03 & 2.78 & 0.32 & 0.19 & 1.190 & 2.145 & 1.059 \\
\hline
\end{tabular}

It is observed that the transport numbers increase with increase in concentration of the electrolytes. This may be due to the fact that with increase in average concentration of the electrolyte, the number of counter ions interacting with the membrane surface increase leading to enhanced Donnan exclusion responsible for increase of transport numbers. The obtained results are in contrast with the Donnan equilibrium theory. The transport numbers tend to stabilize up to a mean concentration of $0.045 \mathrm{M}$. Thereafter, the values of transport numbers and permselectivity 
tend to stay constant. This is due to concentration polarization phenomenon at high concentration resulting in increased co-ion percolation and hence resisting further increase in transport number of cations.

Table 6. Transport numbers at mean concentration for 1:2 electrolytes

\begin{tabular}{|c|c|c|c|c|c|c|c|c|}
\hline \multirow{2}{*}{$\begin{array}{c}\text { Mean concentration } \\
\text { of electrolyte, } \mathrm{M}\end{array}$} & \multicolumn{8}{|c|}{ Transport No. $\bar{t}_{+}$} \\
\hline & $\mathrm{MgCl}_{2}$ & $\mathrm{CaCl}_{2}$ & $\mathrm{SrCl}_{2}$ & $\mathrm{BaCl}_{2}$ & $\mathrm{Mg}\left(\mathrm{NO}_{3}\right)_{2}$ & $\mathrm{Ca}\left(\mathrm{NO}_{3}\right)_{2}$ & $\mathrm{Sr}\left(\mathrm{NO}_{3}\right)_{2}$ & $\mathrm{Ba}\left(\mathrm{NO}_{3}\right)_{2}$ \\
\hline 0.0055 & 1.43 & 1.60 & 1.25 & 2.01 & 1.34 & 1.39 & 1.25 & 1.34 \\
\hline 0.011 & 1.74 & 1.92 & 1.65 & 2.32 & 1.70 & 1.65 & 1.65 & 1.65 \\
\hline 0.0165 & 1.96 & 2.05 & 1.87 & 2.37 & 1.90 & 1.87 & 1.87 & 1.83 \\
\hline 0.022 & 2.09 & 2.2 & 2.0 & 2.45 & 2.0 & 2.0 & 2.00 & 1.87 \\
\hline 0.0275 & 2.18 & 2.27 & 2.09 & 2.50 & 2.18 & 2.05 & 2.09 & 1.92 \\
\hline 0.033 & 2.27 & 2.31 & 2.18 & 2.54 & 2.27 & 2.09 & 2.18 & 1.96 \\
\hline 0.0385 & 2.31 & 2.36 & 2.23 & 2.54 & 2.35 & 2.14 & 2.23 & 2.0 \\
\hline 0.044 & 2.36 & 2.40 & 2.27 & 2.59 & 2.35 & 2.18 & 2.27 & 2.0 \\
\hline 0.0495 & 2.36 & 2.40 & 2.27 & 2.59 & 2.35 & 2.18 & 2.27 & 2.05 \\
\hline 0.055 & 2.35 & 2.36 & 2.18 & 2.56 & 2.31 & 2.16 & 2.18 & 2.02 \\
\hline
\end{tabular}

Fixed charge density

The electrical character of a membrane is expressed in terms of fixed charge density. This fixed charged density of zirconium phosphoborate membrane for 1:1 electrolytes has been evaluated by using Kobatake's equation [30] and is given in Table 7.

Table 7. Values of parameters $\alpha, \beta$ and $\bar{X}$ for zirconium phosphoborate membrane

\begin{tabular}{cccc}
\hline Electrolyte & $\boldsymbol{\alpha}$ & $\beta$ & $\bar{x}$ \\
\hline $\mathrm{LiCl}$ & 0.80 & 2.04 & 0.0075 \\
\hline $\mathrm{NaCl}$ & 0.56 & 4.79 & 0.0078 \\
\hline $\mathrm{KCl}$ & 0.60 & 3.83 & 0.0047 \\
\hline $\mathrm{LiNO}_{3}$ & 0.56 & 4.42 & 0.0072 \\
\hline $\mathrm{NaNO}_{3}$ & 0.68 & 2.55 & 0.0024 \\
\hline $\mathrm{KNO}_{3}$ & 0.62 & 4.34 & 0.0011 \\
\hline $\mathrm{MgCl}_{2}$ & 0.67 & 0.34 & 1.51 \\
\hline $\mathrm{CaCl}$ & 0.69 & 0.23 & 2.22 \\
\hline $\mathrm{SrCl}$ & 0.70 & 0.26 & 1.94 \\
\hline $\mathrm{BaCl}$ & 0.75 & 0.60 & 0.61 \\
\hline $\mathrm{Mg}\left(\mathrm{NO}_{3}\right)_{2}$ & 0.57 & 6.22 & .0036 \\
\hline $\mathrm{Ca}\left(\mathrm{NO}_{3}\right)_{2}$ & 0.57 & 1.15 & 0.49 \\
\hline $\mathrm{Ba}\left(\mathrm{NO}_{3}\right)_{2}$ & 0.62 & 0.76 & 0.67 \\
\hline $\mathrm{Sr}\left(\mathrm{NO}_{3}\right)_{2}$ & 0.61 & 0.13 & 4.69 \\
\hline
\end{tabular}

When negatively charged membrane separates solutions of electrolyte of different concentrations, the membrane potential is given by:

$$
E_{\mathrm{m}}=-\frac{R T}{F}\left\lfloor\frac{1}{\beta} \ln \frac{C_{2}}{C_{1}}-\left(1+\frac{1}{\beta}-2 \alpha\right) \ln \frac{C_{2}+\alpha \beta \bar{X}}{C_{1}+\alpha \beta \bar{X}}\right\rfloor
$$


where $\alpha=\frac{u}{u+v}$ and $\beta=1+\frac{K F \bar{X}}{u}$

$E_{\mathrm{m}}=$ membrane potential difference,

$u$ and $v=$ molar mobilities of cation and anion, respectively,

$K=$ a constant depending on the solution viscosity,

$\bar{X}=$ fixed charged density,

$F=$ faraday constant.

In order to evaluate $\alpha, \beta$ and $\bar{X}$, Kobatake has derived two useful limiting forms, (a) and (b) of equation (3)

a) When $C_{2} \ll<$, equation (3) may be written as:

$$
\left|E_{\mathrm{m}}^{\sigma}\right|=\frac{1}{\beta} \ln \delta-\left(\frac{\delta-1}{\alpha \beta \delta}\right)\left(1+\frac{1}{\beta}-2 \alpha\right) \frac{C_{2}}{\bar{X}}
$$

where, $\left|E_{m}^{\sigma}\right|=$ absolute value of membrane potential given by

$$
\left|E_{m}^{\sigma}\right|=\frac{F E_{\mathrm{m}}}{R T} \text { and } \delta=\frac{C_{2}}{C_{1}}
$$

From the plot of $\left|E_{m}^{\sigma}\right|$ vs. $C_{2}$ in low concentration region, a straight line with an intercept equal to $1 / \beta \ln \delta$ is obtained (Figure 1 ). Thus $\beta$ can be calculated.

b) At fixed $\delta$, inverse of apparent transport number $1 / \bar{t}_{\text {+app }}$ for a coion species in a negatively charged membrane, varies linearly with the inverse of concentration $C_{2}$ at higher electrolyte concentrations, where $1 / \bar{t}_{\text {+app }}$ is defined as

$$
\left|E_{m}^{\sigma}\right|=\left(1-2 \bar{t}_{\text {+app }}\right) \ln \delta
$$

Substituting for $E_{\mathrm{m}}^{\sigma}$ in equation (3) and expanding resultant for expression for $1 / \bar{t}_{\text {+app }}$ in power of $1 / C_{2}$ gives

$$
\frac{1}{\bar{t}_{\text {tapp }}}=\frac{1}{1-\alpha}+\frac{(1+\beta-2 \alpha \beta)(\delta-1) \alpha}{2(1-\alpha)^{2} \ln \delta}\left(\frac{\bar{x}}{C_{2}}\right)
$$

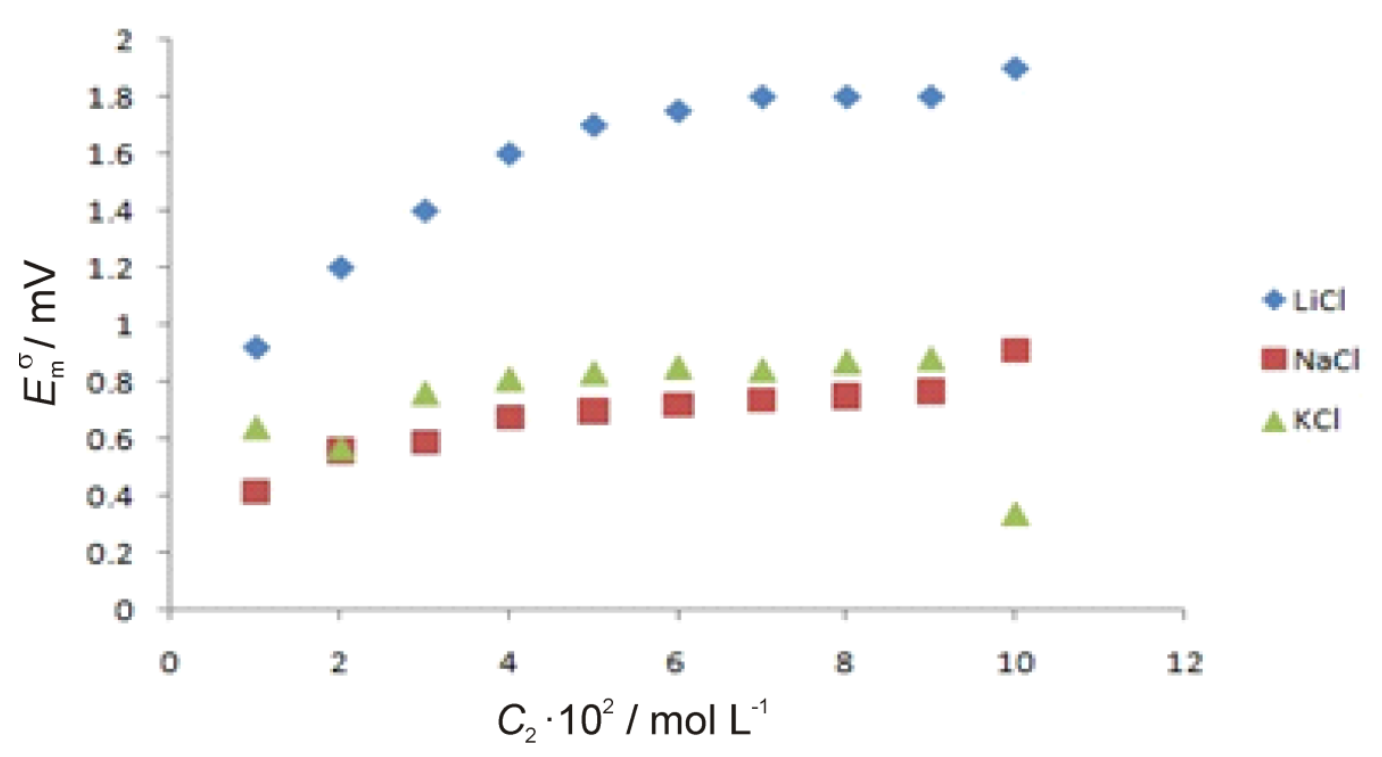

Figure 1. Variation of membrane potential with the concentration of electrolyte 
From equation (3b), it is clear that a plot of $1 / \bar{t}_{\text {tapp }} v s .1 / C_{2}$ (Figure 2 ) at fixed $\delta$ value should be a straight line with an intercept equal to $1 /(1-\alpha)$, from where $\alpha$ can be calculated.

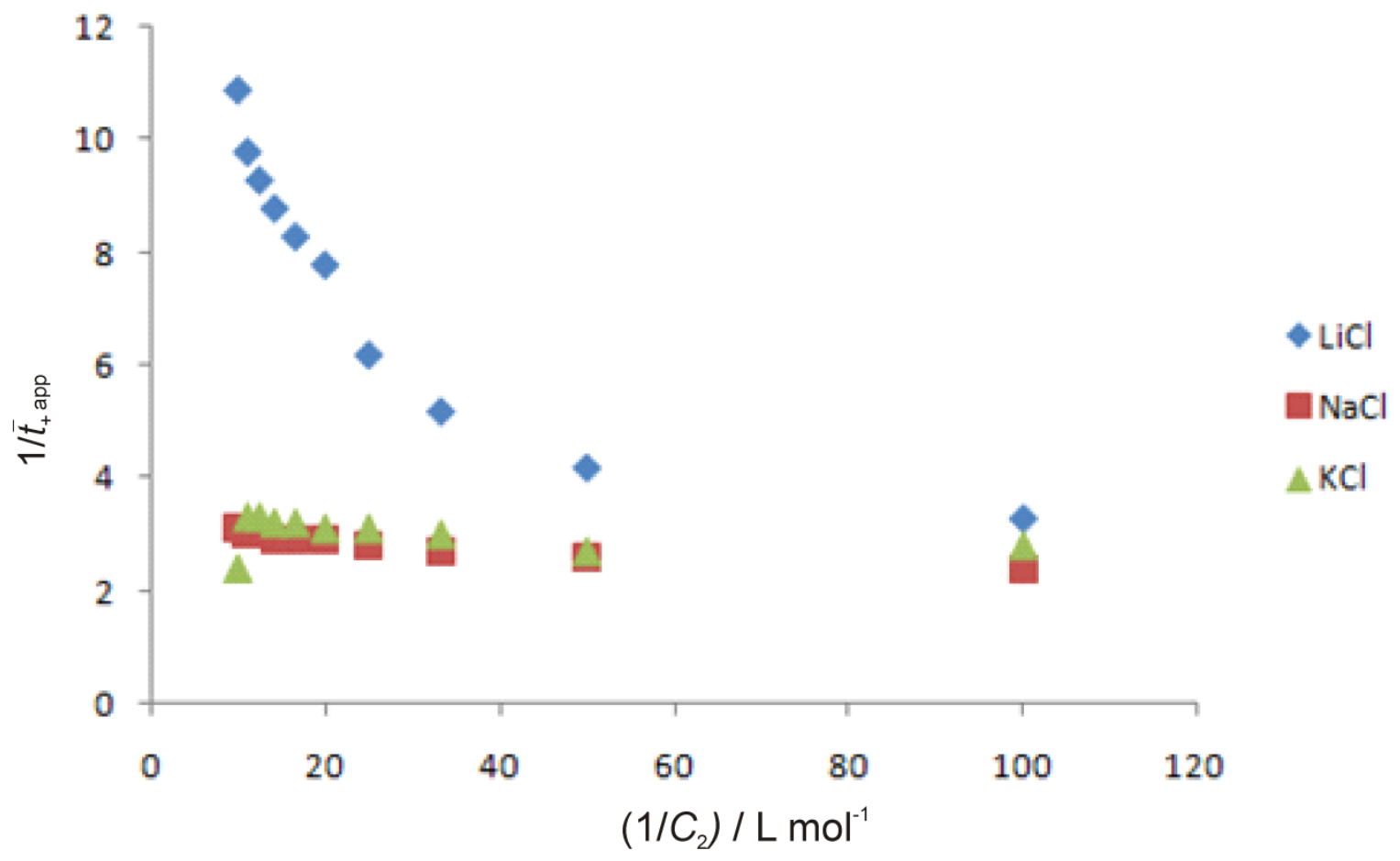

Figure 2. Variation of apparent transport number with reciprocal of concentration

For determining the fixed charge density $\bar{X}$ in dilute concentration range, the value of slope determined from the plot of $\left|E_{\mathrm{m}}^{\sigma}\right|$ vs. $C_{2}$ plot was equated with the slope of equation (3a). $\alpha$ and $\beta$ being known earlier, $\bar{X}$ can be calculated.

$$
\text { Slope }=\frac{\delta-1}{\alpha \beta \delta}\left(1+\frac{1}{\beta}-2 \alpha\right) \frac{1}{\bar{X}}
$$

Apparent transport number $1 / \bar{t}_{\text {+app }}$ indicates transport number of a metal ion in the exchanger whereas the transport number of the same metal ion in solution phase is represented by $t_{+}$. As cited in the literature [31], transport numbers of alkali metal ions in an aqueous system generally increase sharply with initial increase in concentration in low concentration range and then become constant at higher concentration. This is true for all alkali metal ions including $\mathrm{Li}^{+}, \mathrm{Na}^{+}$and $\mathrm{K}^{+}$. As shown in Figure 2, the apparent transport numbers for $\mathrm{Na}^{+}$and $\mathrm{K}^{+}$do not change at all, whereas for $\mathrm{Li}^{+}$, an appreciable linear increase in trend is observed, which indicates that the ion exchanger matrix is selective for some metal ions. The selective behavior of the ion exchange membrane for $\mathrm{Li}^{+}$ions may be either due to steric or electronic reasons. This can't be due to steric reasons because hydrated radii of all the alkali metal ions are almost of the same size. $\mathrm{Li}^{+}$in dehydrated form has large charge to radius ratio as compared to that of $\mathrm{Na}^{+}$and $\mathrm{K}^{+}$.

The different trends of transport number in solution and in membrane phase confirm that the membrane is highly selective for $\mathrm{Li}^{+}$over $\mathrm{Na}^{+}$and $\mathrm{K}^{+}$. This property of the membrane can also be generalized for transition metal ions as well because metal ions of lanthanide series are also different from one another due to their electronic properties while their ionic radii (steric factor) do not change much. 
It is observed that $\bar{t}_{\text {tapp }}$ decreases with increase in mean concentration of the lithium chloride electrolyte. These membrane permeate interactions indicate crystalline morphology [32], being more in amorphous and less in crystalline membranes. The low values indicate very low degree of crystallinity of exchanger material.

The observed values (Table 7 ) of fixed charge density $\bar{X}$ are much lower than those expected from the fixed charge concentration of the exchanger. It indicates that larger part of internal fixed charge remains inactive. It may be due to the reason that active fixed charges in these membranes are essentially those of external surface of grain. As observed in Table 7, the magnitude of $\bar{x}$ values for alkali metal nitrates is lower than for alkali metal chlorides. Hence, the trend in fixed charge density observed for halide and nitrate salts indicates that Donnan exclusion is more applicable for halide salts than for nitrate salts

\section{Conclusions}

The present investigation shows that ion exchange capacity of the ion exchanger decreases with the increase in the radii of hydrated metal ions. The transport numbers increase with increase in average concentration of the electrolyte due to enhanced Donnan exclusion at low electrolyte concentration. The transport numbers decrease at high concentration due to concentration polarization phenomenon. Hence, zirconium phosphoborate membrane shows better characteristics at lower concentrations (up to $0.045 \mathrm{M}$ ) beyond which no appreciable change in activity of the membrane is noticed and remains almost constant. The proposed ion exchange membrane behaves much more selectively for alkaline earth metal ions than alkali metal ions as observed from their respective fixed charge density values.

Acknowledgement: SK and PPS gratefully acknowledge Punjab Technical University (PTU), Jalandhar for permission to work on the project. SKM is thankful to Director, Thapar University, Patiala for the support.

\section{References}

[1] K. G. Varshney, U. Gupta, Bull. Chem. Soc. (Japan) 63 (1990) 1915-1921.

[2] R. D. Noble, S. A. Stern, Membrane Separations Technology: Principles and Applications (Elsevier, Amsterdam), 1995, Chap 7.

[3] M. Ulbricht, K. Richau, H. Kamusewitz, Coll. Surf. A. Physicochem. Eng. Aspects 138 (1998) 353-366.

[4] W. U. Malik, S. A. Ali, Kolloid Z. 175 (1961) 139-144.

[5] W. U. Malik, F. A. Siddiqi, J. Colloid Sci. 18 (1963) 161-175.

[6] W. U. Malik, M. S. Anwar, Indian J. Chem. 3 (1965) 491-496.

[7] W. U. Malik, H. Arif, F. A. Siddiqi, Bull. Chem. Soc. (Japan) 40 (1967) 1746-1753.

[8] W. U. Malik, S. K. Srivastava, P. N. Razdan, S. Kumar, J. Electroanal. Chem. 72 (1976) 111116.

[9] W. U. Malik, S. K. Srivastava, S. P. Arora, R. K. Gulati, J. Electrochem. Soc. (India) 26 (1977) 72-78.

[10] N. P. Gnusin, N. P. Berezina, O. A. Demina, N. A. Kononeko, Russ. J. Electrochem. 32 (1996) 154-163.

[11] S.M. Hosseini, S.S. Madaeni, A.R. Khodabakhshi, Sep. Sci. Technol. 45 (2010) 2308-2321.

[12] P. Dlugolecki, B. Anet, S.J. Metz, K. Nijmejjer, M. J. Wessling, J. Member. Sci. 346 (2010) 163-171.

[13] P. Dlugolecki, K. Nymeijer, S. J. Metz, M. J. Wessling, Membr. Sci. 319 (2008) 214-222.

[14] R. K. Nagarale, G. S. Gohil, V. K. Sahni, R. Rangranjan, Colloids Surf. A 251 (2004) 133-140. 
[15] K. Urano, Y. Masaki, M. Kawabata, Desalination 58 (1986) 171-176.

[16] S. M. Hosseini, S. S. Madaeni, A. R. Khodabakhshi, A. Zendehnam J. Member. Sci, 365 (2010) 438-446.

[17] N. Lakshinarayanaih, Membrane Electrodes, Academic Press, New York, 1976, pp. 50-94.

[18] S. M. Hosseini, S. S. Madaeni, A. R. Khodabakhshi, Sep. Sci. Technol. 46 (2011) 794-808.

[19] S. M. Hosseini, S. S. Madaeni, A. R. Khodabakhshi, J. Member. Sci. 362 (2010) 550-559.

[20] S. M. Hosseini, S. S. Madaeni, A. R. Khodabakhshi, J. Member. Sci. 351 (2010) 178-188.

[21] S. M. Hosseini, S. S. Madaeni, A. R. Khodabakhshi, J. Appl. Polym. Sci. 118 (2010) 33713383.

[22] R.K. Nagarale, V. K. Sahi, S. K. Thampy, R. Rangranjan, React. Funct. Polym. 61 (2004) 131138.

[23] V. K. Shahi, S.K. Thampy, R. Rangaranjan, J. Membr. Sci. 158 (1999) 77-83.

[24] R. K. Nagarale, V. K. Shahi, R. Ragranjan, J. Membr. Sci. 248 (2005) 37-44.

[25] G. S. Gohil, V. V. Bishnu, V. K. Shahi, J. Membr.Sci. 280 (2006) 210-218.

[26] J. Schauer, V. Kuleda, K. Richau, R. Mohr, Desalination 198 (2006) 256-264.

[27] D. R. Lide, CRC Handbook of Chemistry and Physics, 87th Ed., CRC press, Taylor \& Francis Group, Florida (2006-2007).

[28] A. Cimen, M. Ersoz, S. Yildiz, Gazi Uni. J. Sci. 25 (2012) 355-362.

[29] L. Li, J. Dong, T. M. Nenoff, Sep. Purif. Technol. 53 (2007) 42-48.

[30] Y. Kobatake, N. T. Toyoshima, H. Fuzita, J. Phys. Chem. 69 (1965) 3981-3988.

[31] R. L. Balokhra, A. Nag, Indian J. Chem. 32A (1993) 610-615.

[32] R. K. Nagarale, G. S. Gohil, V. K. Shahi, R. Rangrajan, Colloids Surf. A 251 (2004) 133-140.

(C) 2014 by the authors; licensee IAPC, Zagreb, Croatia. This article is an open-access article distributed under the terms and conditions of the Creative Commons Attribution license (http://creativecommons.org/licenses/by/3.0/) (cc) Er 